\title{
Asymptotics for the Boundary Parabolic Anderson Problem in a Half Space*t
}

\author{
René A. Carmona ${ }^{\ddagger}$ and Stanislav A. Grishin \\ Department of Operations Research \& Financial Engineering, \\ Princeton University, Princeton, NJ 08544 \\ and Stanislav A. Molchanov \\ Department of Mathematics \\ University of North Carolina at Charlotte, Charlotte, NC 28223
}

September 16, 2000

\begin{abstract}
We study the large time behavior of the solutions of the Cauchy problem for the Anderson model restricted to the upper half space $D=\mathbb{Z}^{d-1} \times \mathbb{Z}_{+}$and/or $D=$ $\mathbb{R}^{d-1} \times \mathbb{R}_{+}$when the potential is a homogeneous random field concentrated on the boundary $\partial D$. In other words we consider the problem:

$$
\frac{\partial u(t, z)}{\partial t}=\kappa \Delta u(t, z)+\xi(x) u(t, z) \quad z=(x, y), \text { with } y \geq 0 \text { and } t \geq 0
$$

with an appropriate initial condition. We determine the large time asymptotics of the moments of the solutions as well as their almost sure asymptotic behavior when $t \rightarrow \infty$ and when the distance from the boundary, i.e. $y=y(t)$ goes simultaneously to infinity as a function of the time $t$. We identify the rates of escape of $y(t)$ which correspond to specific behaviors of the solutions and different types of dependence upon the diffusivity constant $\kappa$. We also show that the case of the lattice differs drastically from the continuous case when it comes to the existence of the moments and the influence of $\kappa$. Intermittency is proved as a consequence of the large time behavior of the solutions.
\end{abstract}

\footnotetext{
*Running Title: Anderson Problem in a Half Space

${ }^{\dagger}$ AMS Subject Classification: $60 \mathrm{H} 25$

${ }_{\ddagger}^{\ddagger}$ Partially supported by ONR N00014-91-1010
} 


\section{Introduction}

Throughout the paper we study the solutions of the following Cauchy problem, which is known to play a crucial role in many problems appearing in the theory of disordered systems and especially in chemical kinetics and solid state physics (see [10] for details):

$$
\frac{\partial \mathbf{u}(t, z)}{\partial t}=\kappa \Delta u(t, z)+\xi(x) \mathbf{u}(t, z) \quad z=(x, y), t>0
$$

where $x \in \mathbb{Z}^{d-1}, y \in \mathbb{Z}_{+}$in the lattice case and $x \in \mathbb{R}^{d-1}$ and $y \in \mathbb{R}_{+}$in the continuous space. We shall add an initial condition and boundary conditions as needed. We call this problem the Boundary Anderson Problem because the potential $\xi(x)$ is assumed to be a homogeneous random field concentrated on the boundary $\partial D$. This "boundary randomness" is the main originality (and the main thrust) of the paper.

Equation (1) as well as the Hamiltonian $\kappa \Delta+\xi(x)$ which appears in the right hand side of equation (1) have been intensively studied, especially in the lattice case. Most of these studies concern the case of a random potential $\xi(x)$ homogeneous in the whole space (as opposed to a lower dimensional variety as we consider here). In this case, the random self-adjoint operator $\kappa \Delta+\xi(x)$ is known to have almost surely dense pure point spectrum in dimension $d=1$ and for potentials with large fluctuations in all dimensions. These facts from the theory of quantum disordered systems are known under the name of Anderson Localization Theory and are studied mathematically in the framework of the spectral theory of random self-adjoint operators. The interested reader is refered to the book [4] for details on this theory. The spectral theory of operators for which the randomness is restricted to the boundary of the domain is not as advanced. See nevertheless [2].

The unusual spectral properties of random operators have an interesting counterpart in the solutions of the corresponding heat equations. Indeed, their localization properties (i.e. the existence of dense pure point spectrum) translates into the asymptotic intermittency of the family of random fields solving the parabolic equations. This phenomenon was analysed in details in [7] and [10] in the lattice case and in [5] in the continuous models when the potential $\xi(x)$ is a spatially homogeneous (Gaussian and shot-noise Poisson) random field. The present paper is devoted to the analysis of the asymptotics of the solutions of the parabolic equation (1), but when randomness is limited to the boundary of the domain. The main thrust of the paper is twofold: 1) the presence of different asymptotic regimes depending upon the rate at which the distance from the boundary increases, 2) the important differences between the lattice and the continious models which are usually assumed to be similar. 


\subsection{Notations and Assumptions for the Lattice Case}

We consider the equation:

$$
\frac{\partial u(t, z)}{\partial t}=\kappa \Delta u(t, z)+\xi(z) u(t, z) \quad z=(x, y) \in D, t>0
$$

with the initial condition:

$$
u_{0}(0, z)=u_{0}(z), \quad z \in D .
$$

We are mostly interested in the case of a deterministic function $u_{0}(z)$ but we shall also consider the case of a random initial condition $u_{0}$. Here the Laplacian operator $\Delta$ on $D=\mathbb{Z}_{+}^{d}=\mathbb{Z}^{d-1} \times \mathbb{Z}_{+}$is defined as:

$$
\Delta f(z)=\sum_{\left|z-z^{\prime}\right|=1, z^{\prime} \in D}\left[f\left(z^{\prime}\right)-f(z)\right], \quad z \in D
$$

This definition insures that the operator $\Delta$ is self-adjoint with respect to the counting measure. Also, this definition means that a motion driven by the equation (2) is a usual random walk in the interior of $D$ with the following boundary behavior. When a particle is on the boundary, it chooses one of its $2 d$ neighbors with equal probabilities, jumps to this neighbor if the latter is inside $D$ or stays still if jumping would take the particle outside of the domain.

The random potential $\xi(z)$ is assumed to be 0 except on the boundary:

$$
\xi(z)=\xi(x, y)=\xi(x, \omega) \delta_{0}(y)
$$

for a homogeneous random field $\{\xi(x) ; x \in \partial D\}$. We further assume that the $\xi(x)$ are independent and identically distributed random variables. For the distribution of $\xi=\xi(0)$, we only require a mild asymptotic condition on the tail:

$$
\mathbb{P}\{\xi<x\} \sim \exp \left[\frac{-x^{\alpha} L(x)}{2}\right] \quad \text { as } x \rightarrow \infty
$$

for some $\alpha>1$ and for some slowly varying function $L(x)$. Note that the case $\alpha=2$ corresponds to the standart normal distribution; case $\alpha=1$ would correspond to the exponential distribution. We do not consider this case on purpose because the solution of the problem (1) does not exist (almost surely) for the exponentially distributed potential. This fact is a simple consequence of the Feynman-Kac formula. We address the question of existence of solutions in section 2.3. 
Let us remark that, contrary to the homogeneous case treated in [7], we do not require any specific assumptions on the negative part of the potential $\xi(x)$. As it will be seen from the proof, the main contribution to the value of the solution is given by a very special set of trajectories in Feynman-Kac representation (8). In the homogeneous case, the restrictive assumption on the lower tail of the marginal comes from the need to avoid strong negative centers of the potential. In the random boundary model, a particle can easily avoid the regions where the potential is negative by stepping out of the boundary and traveling inside $D$ as needed.

The initial condition $u_{0}(x, y)$ is assumed to be nonnegative and, when it is random, it is assumed to be independent of $\xi$ and homogeneous in $x$ - variable.

\subsection{Notations and Assumptions for the Continuous Case}

We now consider the case of the Euclidean half space $D=\mathbb{R}^{d-1} \times \mathbb{R}_{+}$. The boundary Anderson problem is written in the form of the standard heat equation:

$$
\frac{\partial u(t, z)}{\partial t}=\kappa \Delta u(t, z), \quad z \in D, t>0
$$

with random boundary condition:

$$
\left.\frac{\partial u(t, x, y)}{\partial t}\right|_{y=0}+\xi(x) u(t, x, 0)=0, \quad x \in \mathbb{R}^{d-1}, t>0 .
$$

Here, $\left\{\xi(x) ; x \in \partial D=\mathbb{R}^{d-1}\right\}$ is a homogeneous random field. Notice that the randomness of the medium enters only into the boundary condition but not in the equation (6). This mixed boundary condition (sometimes called Robin condition) is considered to be the best way to mimic the case of the lattice presented in the previous subsection.

The results concerning the models in the whole space $\mathbb{R}^{d}$ (see [5]) suggest that the moment analysis, as well as the almost sure analysis, should be easiest in the case of Gaussian and shot-noise Poisson fields. Unfortunately, the situation is quite different in the random boundary case. Indeed, even though the solution of the parabolic equation does exist for these standard models, we shall show below that these solutions do not have statistical moments. In fact, we shall have to restrict ourselves to potentials having a marginal distribution with smaller tails than the Gaussian tails. Moreover, in order to analyze the large time behavior of the solutions we shall also have to assume that the potential is piecewise constant. A need to impose the additional restrictions on the tails shows that the continuous case is much different from and much more difficult to analyze than the discrete case. This fact is usually denied by most of the workers in the field who consider that the results should be the same in the lattice and in the continuous cases !!! 


\subsection{Existence and Feynman-Kac Representations of the Solutions}

The main purpose of this subsection is to derive the Feynman-Kac representations of the solutions. They read:

$$
u(t, x, y)=\mathbb{E}_{(x, y)}^{(\kappa)}\left\{u_{0}\left(x_{t}, y_{t}\right) \exp \left[\int_{0}^{t} \xi\left(x_{s}, y_{s}\right) d s\right]\right\}
$$

in the lattice case and:

$$
u(t, x, y)=\mathbb{E}_{(x, y)}^{(\kappa)}\left\{u_{0}\left(x_{t}, y_{t}\right) \exp \left[\int_{0}^{t} \xi\left(x_{s}, y_{s}\right) d L_{s}\right]\right\}
$$

in the continuous case. In the former case the expectation is taken over the paths of the continuous time random walk $\left\{z_{t}=\left(x_{t}, y_{t}\right) ; t \geq 0\right\}$ over the lattice $\mathbb{Z}^{d}$ generated by the operator $\kappa \Delta$ defined above (recall the special boundary behavior!). In the latter case, the expectation is taken over the sample paths of the Brownian motion in $D=\mathbb{R}^{d-1} \times \mathbb{R}_{+}$with normal reflection when it hits the boundary $\partial D=\mathbb{R}^{d-1} \times\{0\}$ which we identify to $\mathbb{R}^{d-1}$. This reflected Brownian motion $\left\{z_{t} ; t \geq 0\right\}$ is written in the form $z_{t}=\left(x_{t}, y_{t}\right)$ where $\left\{x_{t} ; t \geq 0\right\}$ is a $(d-1)$-dimensional Brownian motion with variance $\kappa$ and $\left\{y_{t} ; t \geq 0\right\}$ is an independent one dimensional Brownian motion in $\mathbb{R}_{+}=[0, \infty)$ with variance $\kappa$ and reflection at $0 . L_{t}$ is the local time of the $d$-dimensional Brownian motion on the boundary of the domain, and because of the special form of $z_{t}$ it can be identified to the (usual) local time of $y_{t}$ at 0 .

We state the following result, which repeats the corresponding result for the homogeneous case (see [5] and [7]), for the sake of completeness.

Theorem 1.1 Let us assume that the initial condition $u_{0}(z)$ is nonnegative and satisfies:

$$
\limsup _{|z| \rightarrow \infty} \frac{\ln \ln u_{0}(z)}{\ln |z|}<1
$$

almost surely. Then the solutions of problems (2) and (6) exist with probability 1 and are given by the formulas (8) and (9) respectively.

\subsection{Ljapunov Exponents and Intermittency}

We now discuss the notion of intermittency. We adapt a discussion from [10] to the present situation of the half-space $D$ and to the fact that the source of the randomness concentrates on the boundary of the domain. Throughout this subsection we assume that, for each $t>0\left\{X_{r}(x, y(t)) ;(x, y(t)) \in D\right\}$ is a homogeneous (with respect to the space translations of $\partial D$ ) random field on a probability space $(\Omega, \mathcal{F}, \mu)$. We also assume that all the moments $\left\langle\left|X_{r}(x, y(t))\right|^{p}\right\rangle, p=1,2, \ldots$ are finite. We use the notation $\langle X\rangle$ to denote the expectation of the random variable $X$ with respect to the probability $\mu$. 
Definition 1.2 If for some deterministic monotone increasing $A(t)$ (called scale) the limit:

$$
\gamma_{p}=\lim _{t \rightarrow \infty} \frac{1}{A(t)} \ln \left\langle X_{t}^{p}(x, y(t))\right\rangle
$$

exists, then $\gamma_{p}$ is called the p-th moment Ljapunov exponents (with respect to the scale function $A(t)$ ).

This limit does not depend upon the $x$ variable because of the homogeneity assumption. In the case when $X_{t}(x, y(t))=u(t, x, y)$ and $y$ is a constant independent of $t$, the lim in (11) will not depend upon $y$ either and we still can write $\gamma_{p}$ for the $p$-th exponent. We give the definition of intermittency in terms of the moment Ljapunov exponents.

Definition 1.3 The family $\left\{X_{t}(x, y(t)) ;(x, y(t)) \in D\right\}$ of random fields is said to be asymptotically intermittent if:

$$
\gamma_{1}<\frac{\gamma_{2}}{2}<\frac{\gamma_{3}}{3}<\ldots
$$

Notice that wide sense inequalities like (12) hold in full generality because of Hölder's inequality. Moreover these inequalities become strict inequalities as soon as one of them is strict. In other words, intermittency is equivalent to the first enequality of the chain being strict.

Suppose $X_{t}(x, y(t))$ is ergodic in the following sense:

$$
\lim _{Q \uparrow \mathbb{R}^{d-1}} \frac{1}{|Q|} \int_{Q} X_{t}^{p}(x, y(t)) d x=\left\langle X_{t}^{p}(0, y(t))\right\rangle .
$$

Here $|Q|$ denotes the volume of $Q$. The above left hand side is called the $p$-th energy of the the random field $X_{t}(x, y(t))$. The following result (see [7]) illustrates the notion of intermittency.

Proposition 1.4 If the sequence (12) holds true, then the energy of order 2 of $X_{t}(x, y(t))$ concentrates on some random set $E_{t}$ whose measure tends to zero when $t \rightarrow \infty$. In other words, there exists a measurable subset $E_{t}$ of the probability space $\Omega$ such that:

$$
\lim _{t \rightarrow \infty} \mu\left(E_{t}\right)=0
$$

and:

$$
\lim _{t \rightarrow \infty} \frac{1}{\left\langle X_{t}^{2}(x, y(t))\right\rangle}\left\langle X_{t}^{2}(x, y(t)) \mathbb{I}_{E_{t}}\right\rangle=1
$$

where we use the notation $\mathbb{I}_{A}$ for the indicator of the set $A$. 
The result of the above proposition is the mathematical counterpart of the physical intuition behind the notion of intermittency: the overwhelming part of the energy concentrates on systems of 'peaks' the relative area of which going to zero when $t \rightarrow \infty$.

Sometimes it is convenient to use a weaker notion of intermittency.

Definition 1.5 The random family $X_{t}(x, y(t))$ is said to be weakly (asymptotically) intermittent as $t \rightarrow \infty$ if for any $x$ :

$$
\left\langle X_{t}(x, y(t))\right\rangle^{2}=o\left(\left\langle X_{t}^{2}(x, y(t))\right\rangle\right) \quad \text { as } \quad t \rightarrow \infty
$$

The almost sure Ljapunov exponent is defined as follows.

Definition 1.6 If for some scale function a $(t)$ the limit

$$
\gamma=\lim _{t \rightarrow \infty} \frac{1}{a(t)} \ln X_{t}(x, y(t))
$$

exists $\mu$ almost surely, it is called the almost sure Ljapunov exponent with respect to the scale function $a(t)$.

For intermittent random fields the scale function $a(t)$ is usually different from $A(t)$. As in the case of the moment exponents, for $X_{t}(x, y(t))=u(t, x, y)$ with constant $y$ where $u(t, x, y)$ is a solution of (1), $\gamma(y)$ turns out to be independent of $y$. We shall denote it $\gamma$.

\section{Moment Asymptotics in the Lattice Case}

Let $\langle\cdot\rangle$ denotes the expectation with respect to the random media, i.e. the distribution of $\xi$.

Theorem 2.1 Let $z=(x, y) \in D=\mathbb{Z}_{+}^{d}$, let us consider the family of random fields $u(t, x, y)$ solutions of $(2)$ with $u_{0}(x, y) \equiv 1$, and let us set:

$$
n=\frac{\alpha}{\alpha-1}, \quad c=\frac{\alpha-1}{\alpha^{\frac{\alpha}{\alpha-1}} c_{0}^{\frac{1}{\alpha-1}}}
$$

Let $L(x) \equiv c_{0}$ in (5). Then for any $p \geq 1$ there exist three regimes for $\left\langle u^{p}(t, x, y)\right\rangle$ as $t \rightarrow \infty:$

- If $\lim _{t \rightarrow \infty} \frac{y(t)}{t}=0$, then:

$$
\ln \left\langle u^{p}(t, x, y)\right\rangle=c p^{n} t^{n}-p(n-1) y \ln t-\kappa(2 d-1) p t+p \ln \frac{\kappa}{c n p^{n-1}} y+\overline{\bar{o}}(t)
$$




$$
\begin{aligned}
& \text { - If } \lim _{t \rightarrow \infty} \frac{y(t)}{t}=\infty \text { and } \lim _{t \rightarrow \infty} \frac{y(t) \ln t}{t^{n}}<\frac{c p^{n-1}}{n-1} \text { then: } \\
& \ln \left\langle u^{p}(t, x, y)\right\rangle=c p^{n} t^{n}-p(n-1) y \ln t+p \ln \frac{\kappa}{c n p^{n-1}} y-\kappa(2 d-1) p t+\underline{\underline{O}}\left(\frac{y}{t^{n-1}}\right)+\underline{\underline{O}}\left(\frac{y^{2}}{t^{n}}\right)+\overline{\bar{o}}(t) .
\end{aligned}
$$

- If $\lim _{t \rightarrow \infty} \frac{y(t) \ln t}{t^{n}}>\frac{c p^{n-1}}{n-1}$ then:

$$
\left\langle u^{p}(t, x, y)\right\rangle=1+\underline{\underline{O}}\left(e^{-t^{n}}\right)
$$

If $\lim _{t \rightarrow \infty} \frac{y(t) \ln t}{t^{n}}=\frac{c p^{n-1}}{n-1}$ and if $\kappa>c n p^{n-1}$ the regime is (20), if $\kappa<c n p^{n-1}$ the regime is (21), if $\kappa=c n p^{n-1}$ then the regime is determined by the values of the parameters $n, p$, or in other words, by $\alpha$ and $p$. Also, if $0<\lim _{t \rightarrow \infty} \frac{y(t)}{t}<\infty$, the choice between the regimes (19), (20) shoud be made based on the values of the parameters $n, p, \kappa$.

\section{Role of the diffusivity $\kappa$}

The above results illustrate the role of the diffusivity constant $\kappa$ in the asymptotic behavior of the solutions. Under the regime (19) the larger $\kappa$ the smaller the asymptotic moments of the solutions. This type of behavior was typical in the analysis of the homogeneous random media considered in [7] and [10]. The reason can be best understood in terms of the branching random walk. In order to contribute significantly to the value of the solution, particles concentrate near strong potential centers (i.e. the high peaks of the potential). As $t \rightarrow \infty$, these peaks become higher, though at a large distance from each other. The diffusivity $\kappa$ works in two opposite directions: the larger $\kappa$ the easier a particle reaches a strong center. But on the other hand, the larger $\kappa$ the easier the particle leaves this center. The result (19) says that this latter factor is the dominant contribution in the regime under condideration.

Under the regime (20) the situation is just opposite. Distance from the boundary starts playing a role. It takes much longer to reach a strong potential center.

Finally, the regime leading to the asymptotic (21) is simple to understand because the asymptotic behavior of the moments is as if the particle never hits the boundary.

\section{Ljapunov exponents and intermittency}

Theorem 2.1 proves asymptotic intermittency of the family $u(t, x, y(t))$ for the time scale function $A(t)=t^{n}$ in the first two regimes (of course, there is no intermittency in the regime (21)!) Indeed, with this choice of the scale, the moment Ljapunov exponents are given by:

$$
\gamma_{p}=\gamma_{p}(x)=\lim _{t \rightarrow \infty} \frac{1}{A(t)} \ln \left\langle u^{p}(t, x, y)\right\rangle=c p^{n} .
$$


Since we consider only the case $\alpha>1$, we have $n>1$ and consequently:

$$
\frac{\gamma_{p}}{p}>\frac{\gamma_{q}}{q} \quad \text { for } p>q
$$

Note that the property of intermittency is determined by the first term in the asymptotic decomposition (19), which is not affected by changes of $\kappa$.

\section{Random Initial Condition and Assumptions on the Tails}

The result of Theorem 2.1 can be extended in a straightforward manner to the general case of the initial condition which is homogeneous and to the general case of slowly varying function $L(x)$ in the assumption on the tails of the marginals of the potential. The solution still has three different regimes as $t \rightarrow \infty, y=y(t)$ and the intermittency holds for the first two of them.

\section{Potential Distributions}

Theorem 2.1 enlightens the effects of the thickness of the tails of the marginal distributions of the potential. The restriction $\alpha>1$ in (5) corresponds to the condition $n<\infty$ in (18). Its role is to guarantee the almost sure existence of the solution and the existence of the moments. In particular, moments of the solution do not exist if the potential has exponential marginals that corresponds to the case $\alpha=1$. Notice that $n \rightarrow 1$ when $\alpha \rightarrow \infty$. In this regime, we expect that $\gamma_{p} / p$ converges toward a constant. Nevertheless intermittency still holds, in the weak form given in Definition 1.5, as long as marginal distributions are not compactly supported.

\section{Gaussian Distributions}

The Gaussian distributions satisfy condition (5) with $\alpha=2$. In this case we have $n=2, c=1 / 2$, and:

$$
\ln \left\langle u^{p}(t, x, y)\right\rangle=\frac{p^{2} t^{2}}{2}-p y \ln t+p \ln \frac{\kappa}{p} y-\kappa(2 d-1) p t+\underline{\underline{O}}\left(\frac{y^{2}}{t^{2}}\right)+\overline{\bar{o}}(t) .
$$

\section{"Time-dependent Initial Conditions"}

As an easy consequence of the theorem 2.1, we get the following result which treats the special case of initial data depending on time. The asymptotic behavior of the solution is very similar, but the dependence of $u_{0}(x, y)$ upon $t$ affects the second order terms of the formulas. More precisely,

Proposition 2.2 Under the assumptions of Theorem 2.1 if we set $u_{0}(x, y)=\delta_{y_{0}(t)}(y)$, the moments $\left\langle u^{p}(t, x, y)\right\rangle$ have the (logarithmic) asymptotics (19)-(21) with y replaced by $y+y_{0}$. 


\section{The Continuous Case}

We begin this section with two enlightening negative results. They show in a striking manner that the mathematical problems of the continuous case can be very different from the problems and the results of the lattice case. In particular, we show that the frequently used models of random potentials given by homogeneous Gaussian fields and by shot noise homogeneous fields constructed from a Poisson point process are not appropriate as models for boundary potentials because the solutions of the corresponding Cauchy problems fail to have moments!

\subsection{The Case of Gaussian Potentials}

Let us assume that $\left\{\xi(x) ; x \in \mathbb{R}^{d-1}\right\}$ is a homogeneous mean zero Gaussian field with covariance:

$$
\gamma(x)=\langle\xi(x) \xi(0)\rangle
$$

We shall assume that this covariance function $\gamma(x)$ is continuous at the origin. The Gaussian fields form one of the most popular classes of models in the theory of disordered media. See for example [5] for the analysis of the full space. They were included in the analysis of the lattice case (the normal distribution corresponds to $\alpha=2$ ) which we presented in the previous sections.

We are going to show that these fields are not as convenient a model when the randomness appears in the boundary condition. Indeed, in this case, all the moments of the solution to the parabolic problem (6) are infinite.

Theorem 3.1 If the covariance of the homogeneous Gaussian field $\left\{\xi(x) ; x \in \mathbb{R}^{d-1}\right\}$ is continuous at the origin and if the initial condition $u_{0}(x, y)$ is bounded away from 0 on a nonempty open set, then the first moment of the solution $u(t, x, y)$ of $(6)$ is infinite as soon as $t>1 / \gamma(0)$.

Proof:

For the sake of simplicity we assume that the covariance $\gamma(x)$ is nonnegative. The proof of the result in the general situation is more involved and not more instructive. Let us assume for example that:

$$
u_{0}(x, y) \geq \varnothing u>0, \quad x \in U
$$

for some nonempty open set $U$, and let us assume that $t>0$ and $(x, y) \in D$ are fixed. Using the Feynman-Kac representation (9) of the solution $u(t, x, y)$ and Fubini's theorem we get: 


$$
\langle u(t, x, y)\rangle=\mathbb{E}_{(x, y)} u_{0}\left(x_{t}, y_{t}\right)\left\langle\exp \int_{0}^{t} \xi\left(x_{s}, y_{s}\right) d L_{s}\right\rangle
$$

Since for each fixed Brownian path $\xi\left(x_{s}, y_{s}\right)$ is a mean zero Gaussian for each $s, \int_{0}^{t} \xi\left(x_{s}, y_{s}\right) d L_{s}$ is also a mean zero Gaussian random variable. The formula for the Laplace transform gives

$$
\begin{aligned}
\langle u(t, x, y)\rangle & =\mathbb{E}_{(x, y)}\left\{u_{0}\left(x_{t}, y_{t}\right) \exp \left[\frac{1}{2}\left\langle\left(\int_{0}^{t} \xi\left(x_{s}, y_{s}\right) d L_{s}\right)^{2}\right\rangle\right]\right\} \\
& =\mathbb{E}_{(x, y)}\left\{u_{0}\left(x_{t}, y_{t}\right) \exp \left[\frac{1}{2} \int_{0}^{t} \int_{0}^{t} \gamma\left(x_{s}-x_{s^{\prime}}\right) d L_{s} d L_{s^{\prime}}\right]\right\} .
\end{aligned}
$$

Now we use continuity of $\gamma(x)$ at zero. Let us choose $\epsilon \in(0, \gamma(0))$ and let us fix $\delta>0$ small enough so that $|x|<\delta \Rightarrow|\gamma(x)-\gamma(0)|<\epsilon$. Then:

$$
\begin{aligned}
\langle u(t, x, y)\rangle & \geq \mathbb{E}_{(x, y)}\left\{u_{0}\left(x_{t}, y_{t}\right) \exp \left[\frac{1}{2} \int_{0}^{t} \int_{0}^{t} \gamma\left(x_{s}-x_{s^{\prime}}\right) d L_{s} d L_{s^{\prime}}\right] \mathbb{I}\{\mathcal{W}\}\right\} \\
& \geq \varnothing u \mathbb{E}_{(x, y)}\left\{\exp \left[\frac{\gamma(0)-\epsilon}{2} \int_{0}^{t / 2} \int_{0}^{t / 2} d L_{s} d L_{s^{\prime}}\right] \mathbb{I}\{\mathcal{W}\}\right\}
\end{aligned}
$$

where the set $\mathcal{W}=\left\{\sup _{0 \leq s \leq t / 2}\left|x_{s}-x_{0}\right|<\delta,\left(x_{t}, y_{t}\right) \in U\right\}$. Since the expectation is taken over a set of paths which depends only upon the $x$-component of $\left(x_{s}, y_{s}\right)$ and since the local time $L$. depends only upon the $y$-component, we get:

$$
\langle u(t, x, y)\rangle \geq \varnothing u \mathbb{P}_{0}\{\mathcal{W}\} \mathbb{E}_{y} \exp ^{\frac{\gamma(0)-\epsilon}{2}} L_{t / 2}^{2} .
$$

The probability in the right hand side above, say $p_{\delta, t}$, is the probability that a $(d-1)$ dimensional Brownian motion remains inside the ball of radius $\delta / 2$ up to time $t / 2$ and is in $U$ at time $t$. This probability can be estimated from below by:

$$
p_{\delta, t} \geq \frac{c(\delta)}{e^{c_{1}(d) t}}
$$

as $t \rightarrow \infty$ where the constant $c=c(\delta)$ depends also upon the distance between $(x, y)$ and $U$ and $c_{1}(d)>0$. Hence:

$$
\langle u(t, x, y)\rangle \geq \frac{c(\delta)}{e^{c_{1}(d) t}} \mathbb{E}_{y} \exp ^{\frac{\gamma(0)-\epsilon}{2}} L_{t / 2}^{2}
$$


for large time $t$. The remainder of the proof is based on the fact that, when the Brownian path starts from the origin, the distribution of the local time $L_{t}$ is the same as that of the running maximum $M_{t}$. We set $a=\frac{\gamma(0)-\epsilon}{2}$ in order to simplify the notation. Now using the strong Markov property at the stopping time:

$$
\tau_{0}=\inf \left\{s>0, y_{s}=0\right\}
$$

we get:

$$
\begin{aligned}
\langle u(t, x, y)\rangle & \geq e^{O(t)} \mathbb{E}_{y}\left\{e^{a L_{t / 2}^{2}}\right\} \\
& \geq e^{O(t)} \mathbb{E}_{0}\left\{e^{a y_{t / 2-\tau_{0}}^{2}} ; \tau<t / 2\right\} \\
& \sim e^{O(t)} \int_{0}^{t / 2} \int_{-\infty}^{\infty} \exp ^{a y^{2}-\frac{x^{2}}{t-\tau_{0}}} d x \mathbb{P}_{0}\left\{\tau_{0} \in d \tau\right\} .
\end{aligned}
$$

The right hand side of (31) is $\infty$ because the inner integral is equal to $\infty$ for all the values of $t$ satisfying $t>\tau+1 /(a)$ and because the density of the stopping time $\tau$ is strictly positive near 0 .

\subsection{The Case of Poisson Shot-noise Potentials}

The class of shot noise potentials is the second most popular model of random potentials in the theory of random continuous media. We show that, as for the Gaussian potentials, they are ill suited to the problems with random boundary conditions because the corresponding solutions do not have statistical moments.

A shot-noise potential on the boundary is defined as:

$$
\xi(x)=\sum_{i} \varphi\left(x-x_{i}\right) \quad x \in \mathbb{R}^{d-1}
$$

where the points $x_{i}$ form a realization of a homogeneous Poisson point process in $\partial D$ which we identify with $\mathbb{R}^{d-1}$. Let us denote by $\lambda$ the intensity of the point process. We shall assume that the function $\varphi(x)$ (sometimes called the elementary potential) is continuous and strictly positive at zero and satisfies a mild decay property at infinity so that the infinite series defining $\xi(x)$ via formula (32) does converge. See [5] for a detailed discussion of the typical requirements on $\varphi(x)$ in the homogeneous case. The FeynmanKac representation (9) and Fubini's theorem give:

$$
\begin{aligned}
\langle u(t, x, y)\rangle & =\mathbb{E}_{y}\left\{\left\langle\exp \left[\int_{0}^{t} \sum_{i} \varphi\left(x_{s}-x_{i}\right) d L_{s}\right]\right\rangle\right\} \\
& =\mathbb{E}_{y}\left\{\exp \left[\lambda \int_{\mathbb{R}^{d-1}}\left(\exp \left[\int_{0}^{t} \varphi\left(x_{s}-y\right) d L_{s}\right]-1\right) d y\right]\right\}
\end{aligned}
$$


Now we use the continuity of $\varphi(\cdot)$ at zero as we did in the Gaussian case:

$$
\langle u(t, x, y)\rangle \geq \mathbb{E}_{y}\left\{\exp \left[\lambda \int_{\mathbb{R}^{d-1}} \exp ^{(\varphi(0)-\epsilon) L_{t}}-1 d y\right] ; \sup _{0 \leq s \leq t}\left|x_{s}-x_{0}\right| \leq \frac{\delta}{2}\right\}
$$

Again, using the fact that the local time $L_{t}$ has the same distribution as the running maximum $\max _{[0, t]}\left|w_{t}\right|$ when the path starts from the origin, we prove the divergence of the expectation in the right hand side of (34).

\subsection{Some Positive Results}

We saw that the standard (continuous) models of random potentials were not appropriate for the analysis of disorder on the boundary. We now present a model for which the analysis done in the discrete case can be extended to the case of half a Euclidean space. More precisely, we consider a random field $\xi(x, \omega)$ such that:

$$
\gamma(x)=\langle\xi(x) \xi(0)\rangle= \begin{cases}\left\langle\xi^{2}(0)\right\rangle & \text { if }|x|<\delta \\ 0, & \text { otherwise }\end{cases}
$$

and such that condition (5) satisfied together with $L(x) \equiv 1$, which we assume for simplicity of formulas, for some $\alpha>2$. We introduce the parameter:

$$
m=\frac{\alpha}{\alpha-2}
$$

Theorem 3.2 If a random potential $\xi(x)$ satisfies the above conditions, there exist two asymptotic regimes for the solution $u(t, x, y)$ of (6) with $u_{0}(x, y) \equiv 1$ when $t \rightarrow \infty$ :

- If $\lim _{t \rightarrow \infty} y(t) / t^{\frac{m+1}{2}} \leq \frac{a}{b}$ then:

$$
\ln \left\langle u^{p}(t, x, y)\right\rangle=a t^{m}-b y t^{\frac{m-1}{2}}+\overline{\bar{o}}\left(y t^{\frac{m-1}{2}}\right)
$$

- If $\lim _{t \rightarrow \infty} y(t) / t^{\frac{m+1}{2}}>\frac{a}{b}$ then:

$$
\left\langle u^{p}(t, x, y)\right\rangle=1+\overline{\bar{o}}(1)
$$

where $a=\frac{\beta}{2 m} \kappa^{m} c^{\frac{2 m}{\beta}} \beta^{m} p^{2 m}, b=\frac{a m}{\sqrt{2 \kappa a m}}+\frac{\sqrt{2 \kappa a m}}{2 \kappa}(!), \beta=\frac{\alpha}{\alpha-1}$ and $c$ was defined in (18).

This result holds true for more general initial conditions, such as those used in the theorem 3.1 and in the Proposition 2.2. 


\section{The new role of the diffusivity $\kappa$ in the continuous case.}

The diffusivity $\kappa$ appears in the first term of the asymptotics. The reason for this important difference can be explained by a simple argument in terms of a brownian particle.

The proofs of Theorems 2.1 and 3.2 show that the only significant contribution to the solution $u(t, x, y)$ comes from the paths which are well localized in space. More precisely, in the case of the lattice, the first term of the asymptotics is obtained by considering only the paths staying at the same point (potential peak) after they reach this peak. In the continuous case, the main contribution comes from the paths which stay inside a small ball near a high point of the potential. But in the lattice case, when a particle remains at the same point, the role of diffusivity reduces to the probability not to jump. This affects only the lower order terms of the asymptotics. The situation is very different in the continuous case. A particle needs not only to stay inside a small ball, but it also needs to spend some time on the boundary to get a contribution from the local time $L_{t}$ and this new phenomenon is what brings the diffusivity $\kappa$ in the first term. We do not have this effect in the lattice!

\section{Remark on the potential distributions.}

Theorem 3.2 shows that, in the continuous case we still have different regimes for the asymptotic behavior of the solutions. These regimes are similar to those of the lattice case. But the class of distributions for which these asymptotics can be derived is much smaller: $\alpha$ needs to be greater than 2. This is clear because we have $t$ to the power

$$
m=\frac{\alpha}{\alpha-2} \quad \text { instead of } \quad n=\frac{\alpha}{\alpha-1} .
$$

in the leading term of the asymptotic (37).

\section{Almost Sure Asymptotics}

We first consider the lattice case.

Theorem 4.1 Let us assume that the random potential satisfies (5) and that the initial condition $u_{0}(x, y)$ satisfies (10) and (25). Then the solution $u(t, x, y)$ of the problem (2) has the following almost sure logarithmic asymptotics:

- If $\lim _{t \rightarrow \infty} \frac{y(t) \ln f(\ln t)}{t f((d-1) \ln t)}<1$ then:

$$
\begin{aligned}
& \ln u(t, x, y)=t f((d-1) \ln t)-\kappa(2 d-1) t-y \ln f((d-1) \ln t)+y \ln \kappa+\overline{\bar{o}}(t) \\
& \text { - If } \lim _{t \rightarrow \infty} \frac{y(t) \ln f(\ln t)}{t f((d-1) \ln t)}>1 \text { then: }
\end{aligned}
$$

$$
u(t, x, y)=1+\underline{\underline{O}}\left(e^{-t f(\ln t)}\right)
$$


where $f(t)$ is the inverse of the function $t^{\alpha} L(t)$ and $L(t)$ is defined in (5).

The case $\lim _{t \rightarrow \infty} \frac{y(t) \ln f(\ln t)}{t f((d-1) \ln t)}=1$ belongs to either of the regimes depending upon the value of $\kappa:$ if $\kappa>1$ we are in the regime (39), if $\kappa \leq 1$ we are in the regime (40).

The corresponding result in the continuous case is as follows:

Theorem 4.2 The solution $u(t, x, y)$ of the problem (6) with potential satisfying (5) and initial condition satysfying (10) and (25) has the following almost sure logarithmic asymptotics as $t \rightarrow \infty$ :

- If $\lim _{t \rightarrow \infty} \frac{y(t)}{t f(\ln t)} \leq \frac{1}{2} \kappa$ then:

$$
\ln u(t, x, y)=\frac{1}{2} \kappa t f^{2}((d-1) \ln t)-y f((d-1) \ln t)+\overline{\bar{o}}(y f((d-1) \ln t))
$$

- If $\lim _{t \rightarrow \infty} \frac{y(t)}{t f(\ln t)}>\frac{1}{2} \kappa$ then:

$$
u(t, x, y)=1+\overline{\bar{o}}(1)
$$

\section{Role of the diffusivity}

As in the moment analysis in the lattice case, (39) contains two different regimes. The first one, $\lim _{t \rightarrow \infty} \frac{y(t)}{t}=0$, is such that the smaller the diffusivity $\kappa$ the larger the solution. The other regime, $\lim _{t \rightarrow \infty} \frac{y(t)}{t}=\infty$, is such that the larger the diffusivity the larger the solution. The case $0<\lim _{t \rightarrow \infty} \frac{y(t)}{t}<\infty$ belongs to one of these regimes depending on the values of $\kappa$ and $d$. The situation is different in the continuous case. Indeed, the diffusivity always appears in the first term, and an increase of the diffusivity always implies an increase of the statistical moments of the solutions as well as of its almost sure behavior.

\section{Gaussian potential}

In the case of Gaussian potential $\xi(0) \sim N(0,1), f(t)=(2 t)^{1 / 2}$ and

$\ln u(t, x, y)=t \sqrt{2(d-1) \ln t}-\kappa(2 d-1) t-\frac{1}{2} y \ln \ln t+y(\ln \kappa-\ln (2(d-1)))+\overline{\bar{o}}(t)(43)$

for the lattice and

$$
\ln u(t, x, y)=(d-1) \kappa t \ln t-y(t)(2(d-1) \ln t)^{\frac{1}{2}}+\overline{\bar{o}}\left(y(\ln t)^{\frac{1}{2}}\right)
$$

for the continuous cases. 


\section{Proof of the Moment Asymptotics in the Lattice Case}

The proof of the theorem 2.1 is divided into several propositions. First we reduce the problem to the particular case $y=0$. Then we derive the lower bound for $p=1$ and $y=0$. The third step gives the upper bound for this particular case. This step requires the spectral analysis estimates analogous to those given in [10] for the homogeneous case. Next step of the proof comprises the extension of these estimates to higher moments. The last step finishes the proof for the general case $y=y(t) \geq 0$.

The following proposition holds for both, discrete and continuous cases.

\section{Proposition 5.1}

$$
u(t, x, y)=\mathbb{P}\left\{\tau_{0}>t\right\}+\mathbb{E}_{\left(\tau_{0}\right)}\left[u\left(t-\tau_{0}, x, 0\right) ; \tau_{0} \leq t\right]
$$

almost surely with respect to the distribution of $\xi$. The hitting time $\tau_{0}$ was defined in (30). $\mathbb{E}_{\left(\tau_{0}\right)}$ stands for the expectation with respect to the distribution of $\tau_{0}$. Also

$$
\langle u(t, x, y)\rangle=\mathbb{P}\left\{\tau_{0}>t\right\}+\mathbb{E}_{\left(\tau_{0}\right)}\left\langle\left[u\left(t-\tau_{0}, x, 0\right) ; \tau_{0} \leq t\right]\right\rangle
$$

Proof:

(45) is an immidiate implication of the strong Markov property at $\tau_{0}$ in Feynman-Kac formulas (8), (9) and the fact that a particle does not face nonzero potential until $\tau_{0}$. (46) is a consequences of (45) and Fubini's theorem.

Note that (46) can not be directly extended to the higher moments.

The next lemma is computational. It provides the logarifmic asymptotics for the density $p_{\tau_{0}}(t)$ of $\tau_{0}$ for $y=y(t)$.

\section{Lemma 5.2}

$$
\begin{aligned}
& \text { If } \frac{y(t)}{t} \rightarrow \infty \text { then } \\
& \qquad \begin{aligned}
& \ln p_{\tau_{0}}(t) \sim_{t \rightarrow \infty}-y \ln y+y \ln t+y(1+\ln \kappa)-2 \kappa t+\overline{\bar{o}}(t) . \\
\text { If } \lim \frac{y(t)}{t}= & c_{0}<\infty \text { then } \\
\qquad & \ln p_{\tau_{0}}(t) \sim_{t \rightarrow \infty}\left(c_{1}\left(c_{0}\right)-2 \kappa\right) t+\overline{\bar{o}}(t)
\end{aligned}
\end{aligned}
$$

where $c_{1}<2 \kappa$.

Now we derive certain estimates for $\langle u(t, x, 0)\rangle$ leaving the case $y=y(t)>0$ to the end of this section. To get the lower estimate for $\langle u(t, x, 0)\rangle$, we consider only the trajectories of the random walk $\left(x_{s}, y_{s}\right)$ that remain at the starting point until time $t$. 


\section{Proposition 5.3}

$$
\ln \langle u(t, x, 0)\rangle \geq c t^{n}-(2 d-1) \kappa t
$$

Proof:

$$
\langle u(t, x, 0)\rangle \geq\left\langle\mathbb{E}_{(0,0)} \exp ^{\int_{0}^{t} \xi\left(x_{s}, y_{s}\right) d s} ; x_{s}=x_{0}, s \in[0, t]\right\rangle=\left\langle\exp ^{t \xi(0,0)}\right\rangle \mathbb{P}\{N(t)=0\}
$$

where $N(t)$ is the number of jumps before time $t$ provided a particle is on the boundary $\partial D$. $N(t)$ has Poisson distribution with parameter $(2 d-1) \kappa$, and this proves the proposition.

Next proposition reduces the problem (2) to the problem in a bounded domain.

\section{Proposition 5.4}

$$
\langle u(t, x, 0)\rangle \sim_{t \rightarrow \infty}\left\langle\mathbb{E}_{(x, 0)} \exp \left[\int_{0}^{t} \xi\left(x_{s}, y_{s}\right) d s\right] ;\{N(t) \leq t \ln t\}\right\rangle
$$

where $N(t)$ is the number of jumps of the random walk $\left(x_{s}, y_{s}\right)$ started from $(x, 0)$ before time $t$.

Proof:

$$
\mathbb{P}\{N(t) \geq t \ln t\} \leq \mathbb{P}\{\pi(2 d \kappa t) \geq t \ln t\} \sim_{t \rightarrow \infty} \exp [-t \ln t \ln \ln t]
$$

and so

$$
\left\langle\mathbb{E}_{(x, 0)}\left\{\exp ^{\int_{0}^{t} \xi\left(x_{s}, y_{s}\right) d s} ; N(t) \geq t \ln t\right\}\right\rangle \leq \mathbb{E}_{(x, 0)}\left\{\left\langle\exp ^{t \xi(x, 0)}\right\rangle ; N(t) \geq t \ln t\right\} \leq \exp ^{c t^{n}-t \ln t \ln \ln t}
$$

Because of the lower bound (49) it gives (51).

\section{Corollary 5.5}

$$
\langle u(t, x, 0)\rangle \sim_{t \rightarrow \infty}\langle\tilde{u}(t, x, 0)\rangle
$$

where $\tilde{u}(t, x, y)$ is the solution of the problem

$$
\begin{array}{ccc}
\frac{\partial u(t, z)}{\partial t}= & \kappa \Delta u(t, z)+\xi(x) u(t, z) \quad z=(x, y), t \geq 0,(x, y) \in S_{t}^{d+} \\
& u(0, x, y) \equiv 1 \quad S_{t}^{d+}=\{(x, y):|x|<t \ln t, 0 \leq y<t \ln t\}
\end{array}
$$

with zero boundary conditions. 
Problem (55) is a problem in a bounded domain $S_{t}^{d+}$. The evolution operator

$$
H=\kappa \Delta+\xi
$$

has a discrete spectrum $\left\{\mathbb{E}_{k, t}, k=\overline{1, \nu_{t}}\right\}$ and the orthonormal basis $\left\{\psi_{k, t}(x, y)\right\}$. The volume $\nu_{t}$ of $S_{t}^{d+}$ is of order $c t^{d} \ln ^{d} t$, and so

$$
\left\langle\tilde{u}(t, x, 0)^{p}\right\rangle \leq\left\langle\exp \left(p t \max _{k} \mathbb{E}_{k, t}\right)\right\rangle \exp (O(\ln t))
$$

The following proposition gives an upper bound in terms of the greatest eigenvalue $h=h_{t}(a)$ of the operator $H_{0}^{a}=\kappa \Delta+a \delta_{(0,0)}(x, y),(x, y) \in S_{t}^{d+}, a>0$ with zero boundary conditions.

\section{Proposition 5.6}

$$
\left\langle\tilde{u}(t, x, 0)^{p}\right\rangle \leq_{t \rightarrow \infty}\left\langle\exp \left[p t\left(\xi_{(2)}+h_{t}\left(\xi_{(1)}-\xi_{(2)}\right)\right]\right\rangle \exp (O(\ln t))\right.
$$

where

$$
\xi_{(1)}>\xi_{(2)}>\ldots \xi_{\left(\nu_{t}\right)}>
$$

is a variational series of the set of random variables $\left\{\xi(z), z \in S_{t}^{d+}\right\}$

The proof follows from the fact that the upper eigenvalue of $H$ is bounded from above by the upper eigenvalue of the operator $\xi_{(2)}+H_{0}^{\left(\xi_{(1)}-\xi_{(2)}\right)}$.

Lemma 5.7 If $\lim _{t \rightarrow \infty} a(t)=\infty$ then

$$
h_{t}(a)=_{t \rightarrow \infty} a-(2 d-1) \kappa+\frac{c(d, \kappa)}{a}+O\left(\frac{1}{a^{2}}\right)
$$

Proof:

Let $g(x, y)$ be the eigenfunction of $H_{0}^{(a)}$ corresponding to $h_{t}(a)$. We apply Fourier transform to the eigenfunction equation for $g(x, y)$ forth and back to get

$$
g(0,0)=\frac{1}{(n+1)(2 n)^{d-1}} \sum_{\lambda \in \widetilde{S}^{d+}} \exp ^{i(\lambda, z)} \frac{a g(0,0)+g(0,0) \kappa\left(\exp ^{i \lambda_{1}}+1\right)}{h_{t}(a)+2 d \kappa-2 \kappa \sum_{i=1}^{d} \cos \lambda_{i}}
$$

where $\stackrel{\sim}{S}^{d+}=\frac{S^{d+}}{t \ln t}$. Then using Jensen's enequality we get a lower bound of the form:

$$
h_{t}(a) \geq a-(2 d-1) \kappa
$$


Note that, contrary to the homogeneous case, we have $a-(2 d-1) \kappa$ on the right hand side of (62) instead of $a-2 d \kappa$ (ref.[10]). This is due to the fact that nonzero potential $a$ is located on the boundary $\partial D$ and boundary points have only $2 d-1$ neighbors in $D$.

Now we expand the right hand side of (61) in a trigonometric series, which converges for sufficiently big $a$ due to the lower bound (62). The first terms in this expansion constitute formulae (60).

\section{Proposition 5.8}

$$
\left\langle\exp ^{t\left(\xi_{(2)}+h_{t}\left(\xi_{(1)}-\xi_{(2)}\right)\right.}\right\rangle \sim_{t \rightarrow \infty} \exp ^{c t^{n}-(2 d-1) \kappa t}
$$

Proof:

Denote by $p_{t}(x, y)$ the joint density of $\xi_{(1)}, \xi_{(2)}$ and by $p_{\xi}(x)$ the density of $\xi$. Then

$$
p_{t}(x, y)=\nu_{t}\left(\nu_{t}-1\right)[\mathbb{P}\{\xi(0)<y\}]^{\nu-2} p_{\xi}(x) p_{\xi}(y) \sim_{t \rightarrow \infty} p_{\xi}(x) p_{\xi}(y) O(t \ln t),
$$

Using the assumption (5) on the tails of $\xi$ we get

$$
\left\langle\exp ^{t\left(\xi_{(2)}+h_{t}\left(\xi_{(1)}-\xi_{(2)}\right)\right.}\right\rangle \leq \iint_{|x-y|>\delta t^{\frac{1}{\alpha-1}}}+\iint_{|x-y| \leq \delta t^{\frac{1}{\alpha-1}}} \exp ^{\left[t\left(y+h_{t}(x-y)\right)-x^{\alpha}-y^{\alpha}\right]} d x d y
$$

Picking $0<\delta<\frac{1}{\alpha^{\frac{\alpha}{\alpha-1}}}$ we see that the second integral above is asymptotically small (less than $\exp ^{c_{1} t^{n}}$ where $c_{1}<c$ ). Since the quantity we are estimating serves as an upper bound of the solution $u(t, x, 0)$, and because of the lower bound (49), we conclude that only the first integral in (65) is essential. For this integral we use lemma 5.7 to finish the proof.

Proposition 5.8 together with (58) and (50) gives the required upper bound on $\langle u(t, x, 0)\rangle$ and proves theorem 2.1 for $p=1, y=0$.

To prove the general case $p \geq 1, y=0$, we use the following representation of $\left\langle u^{p}(t, x, 0)\right\rangle$ :

$$
\left\langle u^{p}(t, x, 0)\right\rangle=\left\langle\mathbb{E}_{(x, 0)} \exp \left[\sum_{i=1}^{p} \int_{0}^{t} \xi\left(x_{s}^{(i)}, y_{s}^{(i)}\right) d s\right]\right\rangle
$$

where $\left(x_{s}^{(i)}, y_{s}^{(i)}\right)$ - $\mathrm{p}$ independent copies of a random walk $\left(x_{s}, y_{s}\right)$. Consider $\left\langle u^{p}(t, x, 0)\right\rangle$. The lower estimate is immidiate due to (50). It lets us use the problem in a bounded domain $S^{d+}$ instead of the original problem in $D$ to estimate the $p$-th moment of the solution (see the proof of proposition 5.4). Now proposition 5.6 and lemma 5.7 give the desired upper bound. 
Proposition 5.9 If $\lim _{t \rightarrow \infty} \frac{y(t) \ln t}{t^{n}}<\frac{c p^{n-1}}{n-1}$ then:

$$
\begin{gathered}
\ln \left\langle u^{p}(t, x, y)\right\rangle=c p^{n} t^{n}-p(n-1) y \ln t+p \ln \frac{\kappa}{c n p^{n-1}} y-\kappa(2 d-1) p t+\underline{\underline{O}}\left(\frac{y}{t^{n-1}}\right)+\underline{\underline{O}}\left(\frac{y^{2}}{t^{n}}\right)+\overline{\bar{o}}(t) . \\
\text { If } \lim _{t \rightarrow \infty} \frac{y(t) \ln t}{t^{n}}>\frac{c p^{n-1}}{n-1} \text { then: } \\
\left\langle u^{p}(t, x, y)\right\rangle=1+\underline{\underline{O}}\left(e^{-t^{n}} t\right) .
\end{gathered}
$$

Proof:

Only the first asymptotic needs a proof. Using Hölder's inequality we may derive from (45) a lower bound for the higher moments $(p>1)$ of the form

$$
\left\langle u(t, x, y)^{p}\right\rangle \geq\left(\mathbb{E}_{\left(\tau_{0}\right)}\left\langle u\left(t-\tau_{0}, x, 0\right) ; \tau_{0} \leq t\right\rangle\right)^{p}
$$

If we optimize the hitting time at the right hand side of (68) (see the end of the proof of this proposition), we get a lower bound of the form (67) with the only difference that the first term on the right reads $c p t^{n}$ instead of $c p^{n} t^{n}$.

To improve this rough lower bound we use representation (66) to generalize formulae (46) for $\langle u(t, x, y)\rangle$ to the higher moments. Denote by $\tau_{0}^{i}$ the hitting time of $\left(x^{(i)}, y^{(i)}\right)$. Then

$$
\left\langle u^{p}(t, x, y)\right\rangle=\mathbb{E}_{\tau_{0}^{1}} \ldots \mathbb{E}_{\tau_{0}^{p}}\left\langle\prod_{i=1}^{p} u\left(t-\tau_{0}^{i}, x_{s}^{i}, 0\right)\right\rangle
$$

In the last expectation we consider only the indicator of the set of trajectories $\left(x^{(i)}, y^{(i)}\right)$ such that hitting points $\left(x_{\tau_{0}^{i}}^{(i)}, y_{\tau_{0}^{i}}^{(i)}\right)$ are all equal to each other. Since we have the lower bound on $\left\langle u^{p}(t, x, y)\right\rangle$, we use the finite box argument (see proposition 5.4) to show that the asymptotic behavior of $\left\langle u^{p}(t, x, y)\right\rangle$ coincides with itself provided a particle is inside the box of radius, say $\max \left(y, t^{n}\right)$. The size of such a box is asimptotically small, as well as the number of possible combinations for $\left\{x_{\tau_{0}^{i}}^{i}, i=1 \ldots p\right\}$ provided that all of these points belong to the box. This means that we can substitute $x_{s}^{i}$ in (69) by 0 . If we consider the set of trajectories such that the hitting times are close to each other, say $\left\{\tau_{0}^{i} \in B\left(\tau_{0}^{1}, \delta\right), i=1 \ldots p, \delta>0\right\}$ we get

$$
\left\langle u^{p}(t, x, y)\right\rangle \sim \int_{0}^{t}\left\langle u(t-\theta, 0,0)^{p}\right\rangle p_{\tau_{0}}^{p}(\theta) d \theta
$$


which is trivially a lower bound and is also an upper bound because the contribution of all other trajectories is logarifmically insignificant.

(70), lemma 5.2 and the asymptotic representation of $\left\langle u(t, x, 0)^{p}\right\rangle$ (propositions 5.35.8) imply

$$
\left\langle u(t, x, y)^{p}\right\rangle \sim_{t \rightarrow \infty} \exp ^{-p y \ln y+p y(1+\ln \kappa)-(2 d-1) \kappa p t} \int_{o}^{t} \exp ^{c p^{n}(t-\theta)^{n}+p y \ln \theta+\kappa p(2 d-3) \theta} d \theta
$$

The idea is to substitute the integrand by its maximum which is attained for

$$
\theta_{\max } \sim_{t \rightarrow \infty} \frac{y}{c n p^{n-1} t^{n-1}}\left(1+\frac{(n-1) y}{c n p^{n-1} t^{n}}+\underline{\underline{O}}\left(\frac{y^{2}}{t^{2 n}}\right)+\underline{\underline{O}}\left(\frac{1}{t^{2(n-1)}}\right)\right)
$$

This means that for the whole segment, say of the size $\underline{\underline{O}}\left(\frac{y^{2}}{t^{2 n}}+\frac{1}{t^{2(n-1)}}\right)$ we attain a maximum times, of course, some correction which can be estimated. Since the total length of the segment of integration $t$ is logarifmically insignificant and $\underline{\underline{O}}\left(\frac{y^{2}}{t^{2 n}}+\frac{1}{t^{2(n-1)}}\right)$ is logarifmically insignificant in the first regime, the asymptotic behavior of the integral coincides with that of the maximum of the integrand (logarifmically). Computation of the underlined maximum and estimation of all the corrections gives (67) and proves the final result of the theorem 2.1.

\section{Proof of the Almost Sure Asymptotics in the Lattice Case}

We are going to use the following result which is an application of Borel-Cantelli lemma.

Lemma 6.1 Let $\xi_{i}, i=\overline{1, n}$ be independent identically distributed random variables with distribution function satisfying (5). Let $\xi^{(1, n)} \geq \xi^{(2, n)} \geq \ldots \geq \xi^{(n, n)}$ be a variational series of the random sequence $\left\{\xi_{i}, i=\overline{1, n}\right\}$. Then for $n \rightarrow \infty$, for any $0 \leq \delta<1$ almost surely

$$
\begin{gathered}
\max _{n} \xi_{i}=f(\ln n)+\underline{\underline{O}}\left(\frac{\ln \ln n}{(\ln n)^{1-\alpha}}\right) \\
\xi^{\left(n^{\delta}, n\right)}=f((1-\delta) \ln n)+\underline{\underline{O}}\left(\frac{\ln \ln n}{(\ln n)^{1-\alpha}}\right)
\end{gathered}
$$

Proposition 6.2 At the conditions of the theorem 4.1

$$
\ln u(t, x, 0) \geq t f((d-1) \ln t)-\kappa(2 d-1) t+\overline{\bar{o}}(t)
$$

almost surely as $t \rightarrow \infty$. 
Proof:

First consider the case $u_{0} \equiv 1$. Let $B_{(-1)}^{(t)}$ be the ball with center $\left(x_{0}, 0\right)$ and radius $R_{(-1)}^{(t)}=\frac{t}{\ln ^{2} t}$. We count only those paths that stay at the point $\left(x^{*}, 0\right)$, which is chosen to be a maximum of the potential $\xi$ in the ball $B_{(-1)}^{(t)}$, after time 1 .

$$
u(t, x, y) \geq \mathbb{E}_{(0,0)}\left\{\exp ^{\int_{0}^{t} \xi\left(x_{s}, y_{s}\right) d s} ; z_{s}=\gamma_{s}, s \in[0,1] ; z_{1+s}=z_{1}, s \in[0, t-1]\right\}
$$

where $\gamma_{(\cdot)}$ is the shortest path in the interior of $D$ from $\left(x_{0}, 0\right)$ to $\left(x^{*}, 0\right), z=(x, y)$. Thus, $\xi\left(\gamma_{s}\right) \equiv 0$.

Substituting $\xi\left(x_{s}, y_{s}\right)$ by its maximum in the ball $B_{(-1)}^{(t)}$ we use lemma 6.1 to get an almost sure asymptotic of this maximum. Then estimating the length $\left|x^{*}-x_{\tau}\right|$ by the diameter of $B_{(-1)}^{(t)}$, using (52) for $\mathbb{P}\left\{N(1)=\left|x^{*}-x_{\tau}\right|\right\}$ and strong Markov property we finish the proof for the case $u_{0} \equiv 1$.

To prove the general case of $u_{0}(x, y)$ satisfying (25) we should consider the paths which go from $x^{*}$ to $U$ when $t \in[t-1, t]$.

Proposition 6.3 At the conditions of the theorem 4.1

$$
\ln u(t, x, 0) \leq t f((d-1) \ln t)-\kappa(2 d-1) t+\overline{\bar{o}}(t)
$$

almost surely as $t \rightarrow \infty$.

Proof:

Using the lower bound (74) and lemma 6.1 we derive the almost sure 'finite box' argument:

$$
u(t, x, 0) \sim_{t \rightarrow \infty} \tilde{u}(t, x, 0)
$$

almost surely; $\tilde{u}(t, x, 0)$ is the solution of the finite problem (55) in $S_{t \ln t}^{d+}$.

We are going to exploit the fact that

$$
\tilde{u}(t, x, y) \leq \exp ^{t \max _{k} \widetilde{\mathbb{E}}_{k, t}+O(\ln t)}
$$

where $\tilde{\mathbb{E}}_{k, t}$ is the collection of eigenvalues of the operator $\tilde{H}=\kappa \Delta+\widetilde{\xi}(x), x \in S_{t \ln t}^{d+}$ where $\tilde{\xi}(x)=\max \{\xi(x),(1-\epsilon) f((d-1) \ln t)\}$.

Operator $\tilde{H}$ has the following structure:

$$
\tilde{H}=(1-\epsilon) f((d-1) \ln t)+\bar{H}, \quad \bar{H}=\kappa \Delta+\sum_{i=1}^{N_{\epsilon, t}} a_{i} \delta_{x_{i}}(x)
$$


where

$$
\begin{array}{r}
\mathcal{X}_{\epsilon, t}:=\left\{x_{i} \in S_{t \ln t}^{d+}: \xi\left(x_{i}\right)>(1-\epsilon) f((d-1) \ln t)\right\} \\
N_{\epsilon, t}=\#\left\{\mathcal{X}_{\epsilon, t}\right\}, \quad a_{i}=\xi\left(x_{i}, 0\right)-(1-\epsilon) f((d-1) \ln t)
\end{array}
$$

The following lemma is computational. It contains information about the structure of the set $\mathcal{X}_{\epsilon, t}$.

\section{Lemma 6.4}

$$
\begin{gathered}
\max _{i} a_{i} \sim_{t \rightarrow \infty} \epsilon f((d-1) \ln t) \rightarrow \infty \\
N_{\epsilon, t} \sim_{t \rightarrow \infty}\left[\#\left(S_{t \ln ^{2} t}^{d-1}\right)\right]^{\epsilon} \sim t^{\epsilon(d-1)}(\ln t)^{2 \epsilon(d-1)} \\
\min _{1 \leq i \neq j \leq N_{\epsilon, t^{\prime}}}\left|x_{i}-x_{j}\right| \geq_{t \rightarrow \infty}\left(t^{\prime} \ln ^{2} t^{\prime}\right)^{\delta}
\end{gathered}
$$

(a.s) for $\delta<1-2 \epsilon$, where $S_{t^{2}{ }^{2} t}^{d-1}$ is the boundary part of $S_{t \ln ^{2} t^{d+}},\left\{t^{\prime} \rightarrow \infty\right\}$ is a subsequence of $\{t\}$.

The proof of the following lemma, which uses path expansion of the resolvent, can be found in [10].

Lemma 6.5 Let $H=\kappa \Delta+\sum_{i} a_{i} \delta_{x_{i}}(x)$ defined on $L_{2}(S)$ is such that $\inf _{i \neq j}\left|x_{i}-x_{j}\right|=B, a_{i} \geq$ $0, \sup _{i} a_{i}=A$ Then for $\forall \delta>0 \exists A_{0}, B_{0}$ such that for $A>A_{0}, B>B_{0}$ the resolvent $(H-\lambda \mathbb{E})^{-1}$ is analytic in the domain $\lambda \geq A-(2 d-1) \kappa+\delta$, i.e.

$$
S p(H)<A-(2 d-1) \kappa+\delta
$$

Moreover, if the parameters: $|S|, A, B$ are all functions of t, then if

$$
\sum_{n=0}^{\infty} A^{\frac{-n B}{4}} B^{n}|S|^{\frac{n}{2}}
$$

converges, the result (82) is still true.

Note that, as a consequence of lemma 5.2, $2 d-1$ appears at the right hand side of (82).

Lemma 6.4 shows that the conditions of lemma 6.5 are satisfied and so

$$
\max _{k} \overline{\mathbb{E}}_{k, t} \leq A-(2 d-1) \kappa
$$


where $\left\{\overline{\mathbb{E}}_{k, t}\right\}$ denote the collection of eigenvalues of the operator $\bar{H}$. This, together with (76) and (78), implies the upper bound on $u(t, x, 0)$ which coincides with the lower bound (74).

The almost sure behavior of $u(t, x, y)$, as stated in the theorem 4.1 , follows from the formulae (45) and optimization of the hitting time $\tau_{0}$ by the same argument used in the proof of proposition 5.9.

Let us note that the (a.s) existence of the solutions given by (8) and (9) is equivalent to the finiteness of the functionals at the right hand side of these formulas for each $(t, x, y) \in$ $D$ ([7], [12], [14]). Thus the upper bound (75) proves the existence in the lattice case.

\section{Proofs for the Continuous Case}

\subsection{Moment Asymptotics}

\section{Proposition 7.1}

$$
\ln \left\langle u^{p}(t, x, y)\right\rangle \leq \exp ^{a(t-\theta)^{m}} p_{\tau_{0}}(\theta) d \theta
$$

asymptotically when $t \rightarrow \infty$, where $m, a, \beta$ were introduced in the theorem 3.2.

Proof:

The special form of the potential (35) lets us consider the set of points $C=\left\{x_{n}, n=\right.$ $1,2, \ldots\}$ which are the centers of the boxes where potential $\xi(x)$ is constant. Using Feynman-Kac formulae (9) and Hölder's enequality we get

$$
\left\langle u^{p}(t, x, y)\right\rangle \leq\left\langle\mathbb{E}_{(x, y)} \exp ^{p \sum_{x_{n} \in C} \xi\left(x_{n}, 0\right) L_{t}^{\left(x_{n}\right)}}\right\rangle \sim \mathbb{E}_{(x, y)} \prod_{x_{n} \in C} \exp ^{c\left(p L_{t}^{\left(x_{n}\right)}\right)^{\beta}} \leq \mathbb{E}_{(x, y)} \exp ^{c\left(p L_{t}\right)^{\beta}}
$$

where $L_{t}^{\left(x_{n}\right)}$ is the local time a particle spends in the set $\left\{\left|x-x_{n}\right|<\frac{\delta}{2}, y \leq \epsilon\right\}$. The last inequality in the chain (86) is due to the fact that $\beta>1$.

Since the distribution of $L_{t}$ coincides with that of $M_{t-\tau_{0}}^{(\kappa)}$, maximum of a one-dimensional Brownian motion with diffusivity $\kappa$ started from zero, we get

$$
\begin{aligned}
\left\langle u^{p}(t, x, y)\right\rangle \leq \mathbb{E}_{y}^{(\kappa)} \mathbb{E} \exp ^{p M_{t-\tau_{0}}^{(\kappa)}} & \sim \mathbb{E}_{y}^{(\kappa)} \int_{0}^{\infty} \exp ^{c(p x)^{\beta}-\frac{x^{2}}{2\left(t-\tau_{0}\right) \kappa}} d x \\
=\int_{0}^{t} \int_{0}^{\infty} \exp ^{c(p x)^{\beta}-\frac{x^{2}}{2(t-\theta) \kappa}} d x p_{\tau_{0}}(\theta) d \theta & \sim \int_{0}^{t} \exp ^{c\left(p x_{\max }\right)^{\beta}-\frac{x_{\max }^{2}}{2(t-\theta) \kappa} p_{\tau_{0}}(\theta) d \theta}
\end{aligned}
$$


where

$$
x_{\max }=\left(c \beta \kappa p^{\beta}(t-\theta)\right)^{\frac{1}{2-\beta}}
$$

which implies (85).

\section{Proposition 7.2}

$$
\ln \left\langle u^{p}(t, x, y)\right\rangle \geq \exp ^{a(t-\theta)^{m}} p_{\tau_{0}}(\theta) d \theta+\underline{\underline{O}}(t)
$$

asymptotically when $t \rightarrow \infty$.

\section{Proof:}

For the lower bound we need to consider $p$ independent brownian motions, and a representation

$$
\left\langle u^{p}(t, x, y)\right\rangle=\left\langle\mathbb{E}_{(x, y)} \exp \left[\sum_{i=1}^{p} \int_{0}^{t} \xi\left(x_{s}^{(i)}, y_{s}^{(i)}\right) d L_{s}^{(i)}\right]\right\rangle
$$

where $L_{s}^{(i)}$ is the local time at zero of the $i$-th brownian motion $\left(x^{(i)}, y^{(i)}\right)$. Using the argument of proposition 5.9 we get

$$
\left\langle u^{p}(t, x, y)\right\rangle \geq \mathbb{E}_{y}^{(\kappa)}\left\{\mathbb{E}_{(0,0)}\left\langle\exp ^{p L_{t-\tau_{0}}^{(\kappa)} \xi\left(x_{\tau_{0}}\right)}\right\rangle ;\left|x_{s}-x_{\tau_{0}}\right|<\delta / 2, s \in\left[\tau_{0}, t\right]\right\}
$$

Using the fact that the $y$-component of the brownian motion is independent of the $x$-components and the estimate (28) we finish the proof of the proposition as in (87).

Propositions 7.1, 7.2 and a well known formulae for the density $p_{\tau_{0}}(\theta)$ of the hitting time of the brownian motion $\tau_{0}$ imply

$$
\left\langle u^{p}(t, x, y)\right\rangle \sim \int_{0}^{t} \exp ^{a(t-\theta)^{m}-\frac{y^{2}(t)}{2 \theta \kappa}} d \theta \sim \exp ^{a\left(t-\theta_{\max }\right)^{m}-\frac{y^{2}(t)}{2 \theta_{\max } \kappa}}
$$

where

$$
\theta_{\text {max }}=\frac{y}{\sqrt{2 \kappa a m} t^{\frac{m-1}{2}}}\left(1+\frac{(m-1) y}{2 \sqrt{2 \kappa a m} t^{\frac{m+1}{2}}}+\underline{\underline{O}}\left(\frac{y^{2}}{t^{m+1}}\right)\right)
$$

This proves theorem 3.2. 


\subsection{Almost Sure Asymptotic}

Proposition 7.3 At the conditions of the theorem 4.2

$$
\ln u(t, x, 0) \leq \frac{1}{2} \kappa t f^{2}((d-1) \ln t)
$$

almost surely when $t \rightarrow \infty$.

Proof:

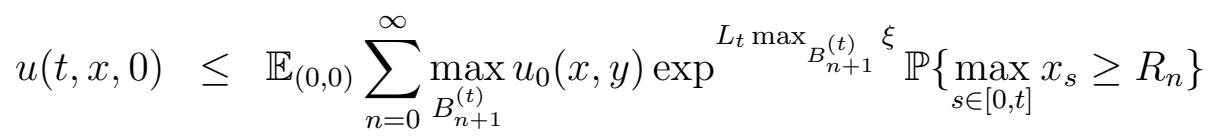

where $B_{n}^{(t)}, n=0,1, \ldots$ is a system of balls in $\mathbb{R}^{d-1}$ with centers at $(x, 0)$ and radiuses $R_{0}=0, R_{1}(t)=t \ln t, \ldots, R_{n}(t)=t \ln ^{n} t, \ldots$

Using the estimate (72) for $\max _{B_{n+1}^{(t)}} \xi$ and the following estimate for the maximum of the $(d-1)$-dimensional brownian motion

$$
\mathbb{P}\left\{\max _{s \in[0, t]} x_{s} \geq R_{n}\right\} \quad \sim_{t \rightarrow \infty} \quad \exp ^{-t \ln ^{n} t}
$$

we conclude that only the first term in decomposition (93) matters and

$$
u(t, x, 0) \leq \mathbb{E}_{(0,0)} \exp ^{L_{t} f((d-1) \ln t)},
$$

asymptotically when $t \rightarrow \infty$. Distribution of $L_{t}$ is the same as that of $M_{t}^{(\kappa)}$ and so

$$
u(t, x, 0) \leq \int_{0}^{\infty} \exp ^{x f((d-1) \ln t)-\frac{x^{2}}{2 t \kappa}} d x
$$

logarifmically. Optimization in $x$ in the integrand gives (92).

Note that formulae (92) proves the existence of the solution in the Feynman-Kac form (9).

Proposition 7.4 At the conditions of theorem 4.2

$$
\ln u(t, x, 1) \geq \frac{1}{2} \kappa t f^{2}((d-1) \ln t)+\underline{\underline{O}}(t)
$$

almost surely when $t \rightarrow \infty$. 
Proof:

The case of compactly supported initial condition $u_{0}(x, y)$ is reduced to the case $u_{0}(x, y) \equiv 1$ as in the proposition 6.2. For $u_{0}(x, y) \equiv 1$, consider a ball $B_{(-1)}^{(t)}$ around point $(x, 1)$ of radius $R_{(-1)}^{(t)}=t^{1-\epsilon}$.

Let us consider the set of paths $\Gamma=\left\{\gamma_{s}:\left|\gamma_{s}-\gamma_{s}^{0}\right|<\frac{\delta}{2}, s \in[0,1]\right\}$, where $\gamma^{0}$ is the path in the interior of $D$ such that the distance $\left|\gamma_{s}^{0}-\partial D\right| \geq \frac{\delta}{2}, s \in[\epsilon, 1]$ for small $\epsilon$, and shortest among those, connecting $x$ and the $\delta$-neighbourhood of $x^{*}$, the center of the plato of maxima of the potential $\xi$ in the ball $B_{(-1)}^{(t)}$. We count only those paths that hit the boundary in the $\delta$-neighbourhood of $x^{*}$ in $\partial D$ and stay in this neighbourhood since then.

$$
u(t, x, y) \geq \mathbb{E}_{y} \mathbb{E}_{0}\left\{\exp ^{\int_{1}^{t} \xi\left(x_{s}, y_{s}\right) d L_{s}^{\prime}} ; z_{s} \in \Gamma, s \in[0,1] ; z_{s} \in O_{\delta}\left(x^{*}\right), s \in[1, t]\right\}
$$

where we used $L_{s}^{\prime}$ instead of $L_{s}$ because the starting point is 1 . However, asymptotically, the distributions of $L_{s}^{\prime}$ and $L_{s}$ are indistinguishable. Using the independence of the components of $\left(x_{s}, y_{s}\right)$, formulae (72) for the maximum of the potential $\max _{B_{-1}^{(t)}} \xi$, estimate (28) for $\mathbb{P}\left\{\left\{z_{s} \in O_{\delta}\left(x^{*}\right), s \in[1, t]\right\}\right.$ and the similar estimate for $\mathbb{P}\left\{z_{s} \in \Gamma, s \in[0,1]\right\}$ we finish the proof of the proposition.

For the case $y=y(t)>0$ we use representation (45) of the solution $u(t, x, y)$ and the fact that the distributions of $\tau_{0}$ and $\tau_{1}$ are asymptotically the same to get the logarifmic asymptotic

$$
u(t, x, y) \sim \int_{0}^{t} \exp ^{\frac{1}{2} \kappa(t-\theta) f^{2}((d-1) \ln (t-\theta))-\frac{y^{2}(t)}{2 \theta \kappa}} d \theta
$$

and then we optimize the integrand in $\theta$ to get (41). Theorem 4.2 is proved.

\section{References}

[1] R.J. Adler (1990): An Introduction to Continuity, Extrema and Related Topics for General Gaussian Processes. Lect. Notes-Monograph Series,12, I.M.S.

[2] M. Aizenman and S. A. Molchanov (1993): Localization at large disorder and extre me energies: an elementary derivation. (preprint).

[3] Ph. Blanchard and Z. Ma (1990): Semigroups of Schródinger Operators with Potentials given by Radon Measures. Stochastic Processes, Physics and Geometry, Albeverio et al, eds, World Scientific.

[4] R. A. Carmona and J. Lacroix (1990): Spectral Theory of Random Schrödinger Operators. Birkhaüser, Boston. 
[5] R. A. Carmona and S. A. Molchanov (1995): Stationary Parabolic Anderson Model and Intermittency. Proba. Theor. Relat. Fields (to appear).

[6] R. A. Carmona and D. Nualart (1993): Gradient Estimates and Parabolic Equations with Random Boundary Conditions. (preprint).

[7] J. Gärtner and S.A. Molchanov (1990): Parabolic Problems for the Anderson M odel. Commun. Math. Phys. 132, 613-615.

[8] I. I. Gihman and A. V. Skorohod (1975): The Theory of Stochastic Processes II, Springer Verlag, New York, N.Y.

[9] J. Glover, M. Rao, H. Sikic and R. Song (1992): Quadratic Forms Corresponding to the Generalized Schrödinger Semigroups. (preprint)

[10] S.A. Molchanov (1994): Lectures on Random Media. Saint Flour Summer School in Probability, Lect. Notes in Math. Springer Verlag (to appear)

[11] S.A. Molchanov and I.D. Novikov (1979): On the Gibbs Free Energy in Quantum Systems. Transl. Moscow Math. Soc. 35, 77-102.

[12] K. Sato and H. Tanaka (1962): Local time on the boundary for multidimentional reflecting diffusion, Proc. Japan Acad. 38, 699-702.

[13] A. S. Sznitman (1993): Brownian asymptotics in a Poisson environment. Prob. Theory Relat. Fields 95, 155-174.

[14] T. Ueno (1967): A Survey on the Markov Process on the Boundary of Multidimentional Diffusion., Proc. Fifth Berkley Sympos. Math. Stat. and Probability, II, Univ. California Berkley, 111-130.

[15] Ya. B. Zel'dovich, S. A. Molchanov, A. A. Ruzmajkin and D. D. Sokolov (1988): Intermittency, diffusion and generation in a nonstationary random medium. Sov. Sci. Rev. Sect. C, Math. Phys. Rev. 7, 1-110. 